\title{
miR-497 suppresses malignant phenotype in non-small cell lung cancer via targeting KDR
}

\author{
YOUYOU XIA ${ }^{1,2^{*}}$, CHENXI HU $^{2 *}$, LIAN LIAN $^{3 *}$, KAIYUAN HUI $^{2}$, LEI WANG $^{2}$, \\ YUN QIAO ${ }^{2}$, LIANG LIU ${ }^{2}$, LIJUN LIANG ${ }^{2}$ and XIAODONG JIANG ${ }^{2}$
}

\begin{abstract}
${ }^{1}$ Department of Oncology, The Affiliated Hospital of Kangda College of Nanjing Medical University, (The First People's Hospital of Lianyungang), Lianyungang, Jiangsu 222002; ${ }^{2}$ Department of Oncology,

The Affiliated Lianyungang Hospital of Xuzhou Medical University, Lianyungang, Jiangsu 222002;

${ }^{3}$ Department of Oncology, Suzhou Xiangcheng People's Hospital, Suzhou, Jiangsu 215131, P.R. China
\end{abstract}

Received July 18, 2018; Accepted April 22, 2019

DOI: $10.3892 /$ or.2019.7163

\begin{abstract}
The aim of the present study was to evaluate the expression of microRNA-497 (miR-497) in non-small cell lung cancer (NSCLC) tissues and cell lines, and to investigate possible mechanisms associated with its regulatory role on cell behaviors. The expression level of miR-497 was evaluated in 15 cases of NSCLC tissues and 8 adjacent normal tissues, and in 8 NSCLC cell lines, including H1975, A549, H358, H1650, H460, Calu-1, H1299 and H292, by reverse transcription-quantitative polymerase chain reaction. Effects of miR-497 overexpression on cell proliferation, invasion, apoptosis and radiosensitivity were examined with a Cell Counting Kit- 8 assay, Matrigel assay, flow cytometry and a clone formation assay in vitro, respectively, and in an in vivo ectopic tumor nude mice model. A dual luciferase reporter assay was employed for interaction between miR-497 and its target gene kinase insert domain receptor (KDR). A significantly decreased level of miR-497 was determined in NSCLC tissues, compared with adjacent normal tissues, and Calu- 1 and H1975 exhibited the lowest miR-497 expression among the 8 NSCLC cell lines. miR-497 overexpression could inhibit cell proliferation and invasion, promote cancer cell apoptosis and decrease cell clone formation following radiation treatment in vitro, and decrease tumor growth in vivo. Furthermore, a dual luciferase reporter assay revealed that KDR as the target gene for miR-497. It was demonstrated that miR-497 was downregulated in NSCLC specimens. Additionally, miR-497 directly targeted and downregulated KDR expression, and inhibited malignant behaviors of NSCLC cells. These data
\end{abstract}

Correspondence to: Dr Xiaodong Jiang, Department of Oncology, The Affiliated Lianyungang Hospital of Xuzhou Medical University, 182 North Tongguan Road, Lianyungang, Jiangsu 222002, P.R. China E-mail: jiangxdlygyy@qq.com

*Contributed equally

Key words: microRNA-497, non-small cell lung cancer, kinase insert domain receptor, radiosensitivity indicated that miR-497 could serve as a tumor suppressor gene involved in NSCLC pathogenesis.

\section{Introduction}

Lung cancer, which possesses the highest incidence of malignant cancer in males, is the most common type of cancer and cause of cancer-associated mortalities in China and globally according to cancer statistics data in 2012 (1). Non-small cell lung cancer (NSCLC) accounts for almost $80 \%$ of lung cancer cases in globally in 2012 (2). Although surveillance and clinical treatment strategies have been improved, the 5-year survival of patients with NSCLC following curative resection is reported to be $30-60 \%$ in globally in 2012 (3). Therefore, elucidating the potential mechanism that mediates the initiation and progression of NSCLC is necessary and of great interest.

MicroRNAs (miRNAs) are a class of small non-coding RNAs with a length of 19-25 bp, which serve an important role in post-transcriptional regulation via target 3 'untranslated region (3'-UTR) (4,5). A single miRNA can target hundreds of mRNAs, and a single mRNA can be coordinately regulated by multiple miRNAs $(4,5)$. Recently, accumulating evidences indicated that miRNAs serve diverse roles in tumorigenesis and cancer progression $(4,5)$. Additionally, miRNAs have received great attention in NSCLC research. A number of deregulated miRNAs in NSCLC, including miR-221, miR-222, miR-449a, miR-21, miR-205, miR-10b, miR-143 and miR-181a, have been demonstrated to regulate cell growth, apoptosis, migration, invasion, angiogenesis, and radiotherapy or chemotherapy resistance (6-8). These observations indicate that deregulation of miRNA expression may be associated with tumorigenesis of NSCLC.

miR-497, a highly conserved miRNA encoded by the first intron of the MIR497HG (Gene ID: 100506755) gene on human chromosome $17 \mathrm{p} 13.1$ (9), is a member of the miR-15/16/195/424/497 gene cluster and has been demonstrated as a tumor suppressor in multiple cancer types, including breast cancer, gastric cancer, colorectal cancer, hepatocellular carcinoma, pancreatic cancer, adrenocortical carcinoma, bladder cancer, melanoma, ovarian cancer, and cervical cancer and other solid tumors (10-13). Zhao et al (14) demonstrated that downregulated miR-497 in NSCLCs could 
result in increased tumor growth and angiogenesis by targeting hepatoma-derived growth factor. However, its role and associated mechanism in NSCLC has not been fully investigated yet. The vascular endothelial growth factor (VEGF) family, an important regulator of angiogenesis, have been demonstrated to serve a critical role in NSCLCs $(15,16)$. However, whether there is an association between miR-497 and molecules in the VEGF family has not been determined.

In the present study, the aim was to evaluate the expression of miR-497 in NSCLC tissues and cell lines, and investigate possible mechanisms associated with its regulatory role on cell behavior.

\section{Materials and methods}

Clinical samples. A total of 15 cases of paraffin-embedded NSCLC tissue (including 8 cases of squamous carcinoma and 7 cases adenocarcinoma; mean age, 58.7 years; age range, $33-75$ years; 10 cases $\geq 60$ years old and 5 cases $<60$ years old; 9 males and 6 females) and 8 cases non-cancerous normal tissue confirmed by a lung needle biopsy were collected from patients without previous radiotherapy or chemotherapy between January 2016 and December 2016 who underwent treatment in the Department of Oncology of the First People's Hospital of Lianyungang (Lianyungang, China). The exclusion criteria included: Patients who had received chest radiotherapy or systemic chemotherapy prior to sampling; previous history of oncology; participation in other drug clinical trials and anti-tumor treatments; pregnant and lactating women; and known history drug abuse (except alcohol abuse). All the samples were from human lung biopsy tissue and confirmed by experienced pathologists who were blinded to the study. The present study was approved by the ethics committee of the First People's Hospital of Lianyungang. Written informed consent was obtained from the participating individuals.

Cell culture. The human NSCLC cancer cell line Calu-1 (human squamous cell carcinoma) (17), H358 (human lung adenocarcinoma, \#SCSP-583) (18), H460 (human lung large-cell carcinoma) (19), H292 (human lung mucoepidermoid carcinoma-lymph node metastatic strain) (20), H1650 (human lung adenocarcinoma) (21), A549 (human lung adenocarcinoma) (22), H1975 (human lung adenocarcinoma) (21), and H1299 (human lung adenocarcinoma lymph node metastatic strain) (23) were obtained from the Cell Bank of Shanghai Institute of Biological Science, Chinese Academy of Science (Shanghai, China). Among these cell lines, Calu-1 was cultured with McCoy's 5A medium containing $10 \%(\mathrm{v} / \mathrm{v})$ fetal bovine serum and $1 \%$ antibiotics, while other cell lines were maintained as monolayers when cultured in cell culture flasks with RPMI-1640 medium containing 10\% (v/v) fetal bovine serum and $1 \%$ antibiotics. Cells were cultured at $37^{\circ} \mathrm{C}$ in a humidified atmosphere containing $5 \% \mathrm{CO}_{2}$. All the cell culture medium and additives were purchased from Invitrogen (Thermo Fisher Scientific, Inc., Waltham, MA, USA). All 8 cell lines were used for expression analysis miR-497 by reverse transcription-quantitative polymerase chain reaction (RT-qPCR), whereas Calu-1 and H1975 cells were further employed for the cell proliferation, cell migration, cell apoptosis, cell radiosensitivity, miR-497 and KDR interaction analysis, and the in vivo tumor formation experiment.
Cell transfection. miR-497 mimic and miR-NC were obtained from the Sangon Biotech Co., Ltd. (Shanghai, China) and the detailed information regarding the sequence were from previous description (24). Cells were grown to a confluence of $\sim 40 \%$ and transfected with $50 \mathrm{nM}$ miR-mimic and miR-NC encapsulated with Lipofectamine ${ }^{\circledR} 2000$ (Invitrogen; Thermo Fisher Scientific, Inc.) in Gibco Opti-MEM ${ }^{\mathrm{TM}}$ I Reduced Serum medium (cat. no. 31985-062; Gibco; Thermo Fisher Scientific, Inc.) for $48 \mathrm{~h}$. All the experiments were repeated for at least 3 times.

$R T-q P C R$. Total NSCLC tissue RNA or cellular RNA of human NSCLC cancer cell lines were extracted using TRIzol ${ }^{\circledR}$ reagent (Invitrogen; Thermo Fisher Scientific, Inc.). RT-qPCR for kinase insert domain receptor (KDR) was conducted using an One Step SYBR ${ }^{\circledR}$ PrimeScript ${ }^{\mathrm{TM}}$ RT-PCR kit (Takara Biotechnology Co., Ltd., Dalian, China) and an iQ5 Real-time PCR Detection system (Bio-Rad Laboratories, Inc., Hercules, CA, USA). The PCR conditions were 1 cycle for $30 \mathrm{sec}$ at $95^{\circ} \mathrm{C}, 40$ cycles of $5 \mathrm{sec}$ at $95^{\circ} \mathrm{C}$ and $34 \mathrm{sec}$ at $60^{\circ} \mathrm{C}$, followed by 1 cycle for $15 \mathrm{sec}$ at $95^{\circ} \mathrm{C}$ and $60 \mathrm{sec}$ at $60^{\circ} \mathrm{C}$. Expression of the GAPDH gene was assessed simultaneously in all samples as an internal control. For miRNA RT-qPCR, Taqman ${ }^{\circledR}$ MicroRNA Reverse Transcription and Taqman MicroRNA Assay (Applied Biosystems; Thermo Fisher Scientific, Inc.) kits were employed for experiments. The expression of U6 was used as the internal control. Relative gene expression was determined with the $2^{-\Delta \Delta \mathrm{Cq}}$ method (25). Oligonucleotide primers specific for KDR and GAPDH were obtained from Sangon Biotech Co., Ltd. The sequences of the PCR primers used are as follows: KDR, 5'-CCGTCAAGGGAAAGACTA CG-3' (forward) and 5'-AGATGCTCCAAGGTCAGGAA-3' (reverse); miR-497, 5'-GTGCAGGGTCCGAGGT-3' (forward), and 5'-TAGCCTGCAGCACACTGTGGT-3' (reverse); and U6, 5'-GCTTCGGCACATATACTAAAA-3' (forward), and 5'-CGCTTCACGAATTTGCGTGTCAT-3' (reverse).

Cell proliferation. Human NSCLC cancer cell lines Calu-1 and H1975 (3x10 3 cells) were seeded in 96-well plates in complete medium [Calu-1 cells were cultured with McCoy's 5A medium containing $10 \%(\mathrm{v} / \mathrm{v})$ fetal bovine serum and $1 \%$ antibiotics, H1975 cells were maintained as monolayers when cultured in cell culture flasks with RPMI-1640 medium containing 10\% (v/v) fetal bovine serum and $1 \%$ antibiotics] and transfected with miR-497 mimic and miR-NC, as aforementioned. After 2 days at $37^{\circ} \mathrm{C}, 5 \% \mathrm{CO}_{2}$, cell proliferation during a time period of consecutive 4 days was evaluated by Cell Counting Kit- 8 method (Dojindo Molecular Technologies, Inc., Kumamoto, Japan), according to the manufacturer's protocols, using a microplate reader (Molecular Devices LLC, Sunnyvale, CA, USA) to measure the absorbance.

Cell apoptosis. Human NSCLC cancer cell lines Calu-1 and H1975 (800 cells) were seeded in 6-well plates in complete medium [Calu-1 cells were cultured with McCoy's 5A medium containing $10 \%(\mathrm{v} / \mathrm{v})$ fetal bovine serum and $1 \%$ antibiotics, H1975 cells were maintained as monolayers when cultured in cell culture flasks with RPMI-1640 medium containing $10 \%$ (v/v) fetal bovine serum and $1 \%$ antibiotics] and transfected with miR-497 mimic and miR-NC, as aforementioned. After medium replacement [Calu-1 cells were cultured with McCoy's 
$5 \mathrm{~A}$ medium containing $10 \%(\mathrm{v} / \mathrm{v})$ fetal bovine serum and $1 \%$ antibiotics, $\mathrm{H} 1975$ cells were maintained as monolayers when cultured in cell culture flasks with RPMI-1640 medium containing $10 \%(\mathrm{v} / \mathrm{v})$ fetal bovine serum and $1 \%$ antibiotics] at $48 \mathrm{~h}$ post-transfection, the cells were irradiated with $2 \mathrm{~Gy} \mathrm{X}$ ray to efficiently induce apoptosis within $48 \mathrm{~h}$, and then harvested and suspended for the assay. Subsequently, $\sim 1 \times 10^{7}$ cells were washed in PBS, resuspended in Annexin $\mathrm{V}$ and propidium iodide (PI) staining solution containing $5 \mu \mathrm{l}$ Annexin V, $10 \mu \mathrm{l}$ PI and $195 \mu \mathrm{l}$ binding buffer (Annexin V-FITC/PI Double Dyeing Cell Apoptosis Test kit; Nanjing KeyGen Biotech Co., Ltd., Nanjing, China) and incubated at room temperature for 20 min under the dark condition, and then analyzed immediately with a flow cytometer (BD FACS Aria) by BD FACSDiva software version 4.0 (BD Biosciences; Becton, Dickinson and Company, Franklin Lakes, NJ, USA).

Clone formation. Human NSCLC cancer cell lines Calu-1 and H1975 (800 cells) were seeded in 6-well plates in complete medium [Calu-1 cells were cultured with McCoy's 5A medium containing $10 \%(\mathrm{v} / \mathrm{v})$ fetal bovine serum and $1 \%$ antibiotics, while other cell lines were maintained as monolayers when cultured in cell culture flasks with RPMI-1640 medium containing $10 \%(\mathrm{v} / \mathrm{v})$ fetal bovine serum and $1 \%$ antibiotics] and transfected with miR-497 mimic and miR-NC, as aforementioned. After medium replacement [Calu-1 cells were cultured with McCoy's 5A medium containing $10 \%$ (v/v) fetal bovine serum and $1 \%$ antibiotics, H1975 cells were maintained as monolayers when cultured in cell culture flasks with RPMI-1640 medium containing $10 \%(\mathrm{v} / \mathrm{v})$ fetal bovine serum and $1 \%$ antibiotics] at $48 \mathrm{~h}$ post-transfection, the cells were irradiated with a series dose of 0-8 Gy X-rays and maintained at $37^{\circ} \mathrm{C}$ in a humidified atmosphere containing $5 \% \mathrm{CO}_{2}$ for 12 days, when they were stained with crystal violet at room temperature for $1 \mathrm{~h}$. The colony survival (a colony was defined as $\geq 50$ cells) was counted under a light microscope (x40; ECLIPSETs2; Nikon Corporation, Tokyo, Japan). The whole process was performed three times to obtain the mean number of colony formations.

Cell invasion. A Transwell system was employed to perform the cell invasion assay. Briefly, resuspended Calu-1 and H1975 cells [Calu-1 cells were cultured with McCoy's 5A medium containing $10 \%$ (v/v) fetal bovine serum and 1\% antibiotics, H1975 cells were maintained as monolayers when cultured in cell culture flasks with RPMI-1640 medium containing $10 \%$ (v/v) fetal bovine serum and $1 \%$ antibiotics] $\left(2 \times 10^{5}\right.$ cells) transfected with miR-497 mimic and miR-NC, as aforementioned, were seeded into the upper chamber prefilled with Matrigel, and the lower chamber was added with RPMI-1640 medium supplemented with $20 \%$ FBS. After the transwell plate was maintained in a routine cell culture incubator for a specific period of time of $48 \mathrm{~h}$, the upper chamber was retained and the membranes were obtained for hematoxylin staining at room temperature for 1 min. The cell number of each membrane was determined in 3 randomly picked fields (x200 magnification) under a light microscope. All the experiments were performed in triplicate.

Western blotting. Cells obtained from the aforementioned treatment (routine cell culture or after transfection with miR-497 mimic and miR-NC) were lysed in radioimmunoprecipitation buffer (Beyotime Institute of Biotechnology, Beijing, China), followed by high-speed centrifugation $(15,000 \mathrm{x}$ g for $5 \mathrm{~min}$ at $4^{\circ} \mathrm{C}$ ) and protein quantification using a bicinchoninic acid assay. Cellular proteins $(20 \mu \mathrm{g})$ were separated by $10 \%$ SDS-PAGE and transferred onto polyvinylidene difluoride membranes. Following blocking in 5\% non-fat milk containing PBS at room temperature for $2 \mathrm{~h}$, the membranes were incubated with anti-VEGF receptor 2 (VEGFR2) monoclonal primary antibodies (1:1,000 dilution; Cell Signaling Technology, Inc. Danvers, MA, USA; \#9698) at room temperature for $1 \mathrm{~h}$. GAPDH (1:1,000 dilution; Santa Cruz Biotechnology, Inc., Dallas, TX, USA; \#sc-32233) was used at room temperature for $1 \mathrm{~h}$ as the loading control. Appropriate horseradish peroxidase-conjugated secondary antibodies (anti-rabbit IgG, HRP-conjugated antibody; 1:2,000 dilution; incubated at room temperature for $1 \mathrm{~h}$; Cell Signaling Technology, Inc.; cat. no. 7074) were applied to detect labeled proteins. The protein bands were developed with a SuperSignal Ultra Chemiluminescent Substrate (Pierce; Thermo Fisher Scientific, Inc.) on X-ray films (Kodak, Rochester, NY, USA).

Dual luciferase reporter assay. The possible miR-497 binding sites in KDR gene 3'-UTR were predicted using bioinformatics software (Targetscan version 7.1; http://www. targetscan.org/vert_71/). The predicted and mutated sequences targeting on KDR 3'-UTR were amplified and cloned into a pMIR-REPORT Dual-Luciferase miRNA Target Expression Vector (Promega Corporation, Madison, WI, USA; named pMIR-REPORT-KDR-WT and pMIR-REPORT-KDR-MUT, respectively). Subsequently, Calu-1 and H1975 cells were co-transfected with $0.5 \mu \mathrm{g}$ pMIR-REPORT-KDR-WT or pMIR-REPORT-KDR-MUT vectors, and $100 \mathrm{nM}$ negative control (NC) miRNA or miRNA-mimics (Shanghai GenePharma Co., Ltd., Shanghai, China) using Lipofectamine 2000. The luciferase activities were measured using a Dual-Luciferase Reporter Assay kit (Promega Corporation) $48 \mathrm{~h}$ after transfection. A control reporter was co-transfected to provide an internal control for normalizing the activity of the experimental reporter.

Lentivirus mediated miR-497 knockdown. Recombinant lentiviral particles expressing miR-497 (5'-CAGCAGCACACU GUGGUUUGUA-3') or scramble control siRNA (5'-UUCUCC GAACGUGUCACGUTT-3') were obtained from Shanghai GenePharma Co., Ltd. Cells were grown to $40 \%$ confluence and transduced with complete medium [Calu-1 cells were cultured with McCoy's 5A medium containing $10 \%(\mathrm{v} / \mathrm{v})$ fetal bovine serum and $1 \%$ antibiotics, and H1975 cells were maintained as monolayers when cultured in cell culture flasks with RPMI-1640 medium containing $10 \%(\mathrm{v} / \mathrm{v})$ fetal bovine serum and $1 \%$ antibiotics] containing lentiviral particles expressing miR-497 or scramble control siRNA at concentrations of $1 \times 10^{8}$ transducing units/ml [multiplicity of infection (MOI) of 20] (18) at $37^{\circ} \mathrm{C}$ for 48 h. Polybrene (cat. no. H9268; Sigma-Aldrich; Merck KGaA, Darmstadt, Germany) at a concentration of $8 \mu \mathrm{g} / \mathrm{ml}$ was added simultaneously to increase infection efficiency. No adverse effects were observed on the cell viability by siRNA or Polybrene (data not shown). The siRNAs had no off-target effects, and did not affect cell adherence, shape and viability at the MOI of 20, according to manufacturer's protocol and treatment duration. The cells were used at $48 \mathrm{~h}$ after infection. 


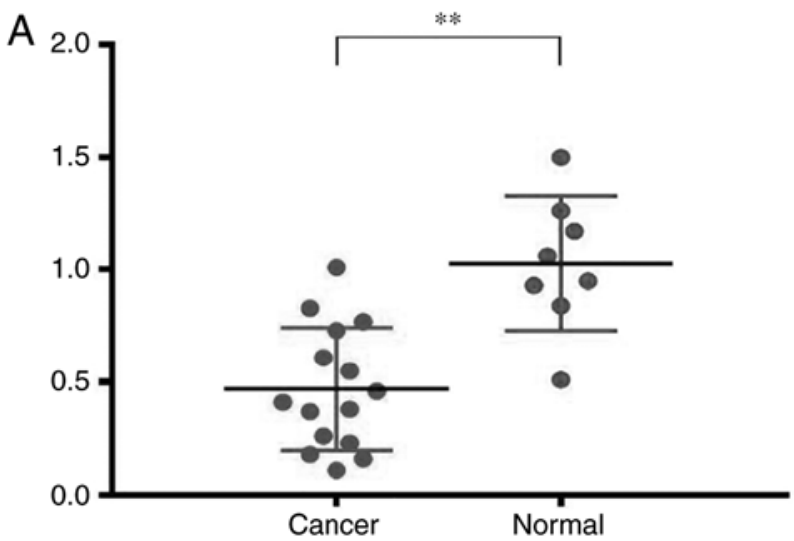

\begin{tabular}{|c|c|c|}
\hline & Cancer & Normal \\
\hline Mean & 0.47 & 1.03 \\
\hline SD & 0.27 & 0.27 \\
\hline & Cancer & Normal \\
\hline \multicolumn{3}{|l|}{ Number of points } \\
\hline \#Y values analyzed & 15 & 8 \\
\hline Outliers & 0 & 0 \\
\hline
\end{tabular}

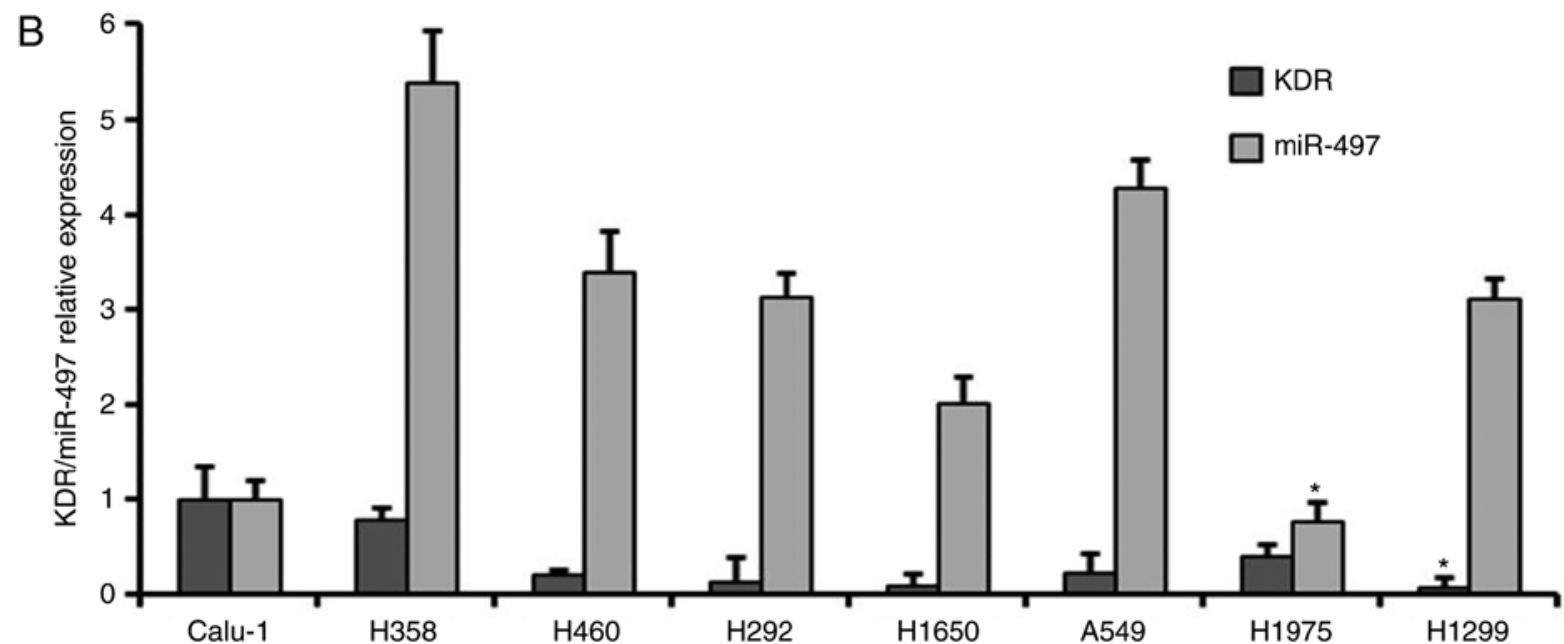

Figure 1. Reverse transcription-quantitative polymerase chain reaction evaluation of the expression level of miR-497 in NSCLC tissues and cell lines. (A) A total of 15 paraffin-embedded NSCLC tissues and 8 non-cancerous adjacent normal tissues were collected for miR- 497 level evaluation. Significantly decreased miR-497 level was determined in NSCLC tissues, compared with the control tissues. (B) A total of 8 NSCLC cell lines, including H1975, A549, H358, H1650, H460, Calu-1, H1299 and H292, were collected for miR-497 level evaluation. Calu-1 and H1975 cells were confirmed as having the lowest two expression levels of miR-497 among the 8 cell lines. " $\mathrm{P}<0.05$, compared with the other cell lines. ${ }^{* * *} \mathrm{P}<0.01$. NSCLC, non-small cell lung cancer; miR, microRNA; KDR, kinase insert domain receptor.

Nude mice model of ectopic tumor. Athymic nude $(\mathrm{nu} / \mathrm{nu})$ mice (male:female, 1:1 ratio; total 8 mice) at 6 weeks old (20-22 g) were purchased from Shanghai SLAC Laboratory Animal Co., Ltd. (Shanghai, China), housed in an air-conditioned room at $22^{\circ} \mathrm{C}$ with a $12 / 12 \mathrm{~h}$ light/dark cycle and $40-60 \%$ humidity and free access to food and water. The tumors were generated by subcutaneous injection of $2 \times 10^{6} \mathrm{miR}-497$ or scramble control siRNA lentivirus particles infected Calu-1 cell suspended in $50 \mu 1$ PBS into the dorsal region near the thigh. Mice were then weighed and assessed for tumor size every three day by measuring tumor length and tumor width. Furthermore, the body weight of the mice was also determined every 5 days. At week 4 post-treatment, all the mice were sacrificed by cervical dislocation and the tumors were excised, weighed and imaged. The study protocol was approved by the Institutional Animal Care and Use Committee of the First People's Hospital of Lianyungang, which is adherent to the generally accepted international guidelines for animal experimentation (26).

Statistical analysis. All statistical analyses were conducted using SPSS v18 (SPSS, Inc., Chicago, IL, USA) and GraphPad Prism 7.0 (GraphPad Software Inc., San Diego, CA, USA).
Data are presented as the mean \pm standard deviation. The unpaired Student's t-test or one-way analysis of variance followed by Tukey's post hoc test was used to examine differences between groups. Dose-response cell survival curves were fitted to a multitarget model, $S=1-(1-e-D / D 0)^{\mathrm{N}}$. S, ratio of survival tumor cells at a given dose; D, a given dose; D0, the dose increment that reduces the cell survival to $37 \%$ of the initial value depicted on the exponential portion of the curve; $\mathrm{N}$, back extrapolation of the exponential portion of the survival curve to zero dose. $\mathrm{P}<0.05$ was considered to indicate a statistically significant difference.

\section{Results}

Expression of miR-497 in NSCLC tissues and cell lines. In order to investigate the role of miR-497 in NSCLC, the expression level of miR-497 was examined in 15 NSCLC tissues and 8 non-cancerous adjacent normal tissues, and the results demonstrated that significantly decreased levels of miR-497 expression in NSCLC, compared with normal tissues $(0.47 \pm 0.27$ vs. $1.03 \pm 0.27 ; \mathrm{P}<0.05$; Fig. $1 \mathrm{~A})$. Furthermore, the expression of miR-497 and KDR in 8 NSCLC cancer cell 

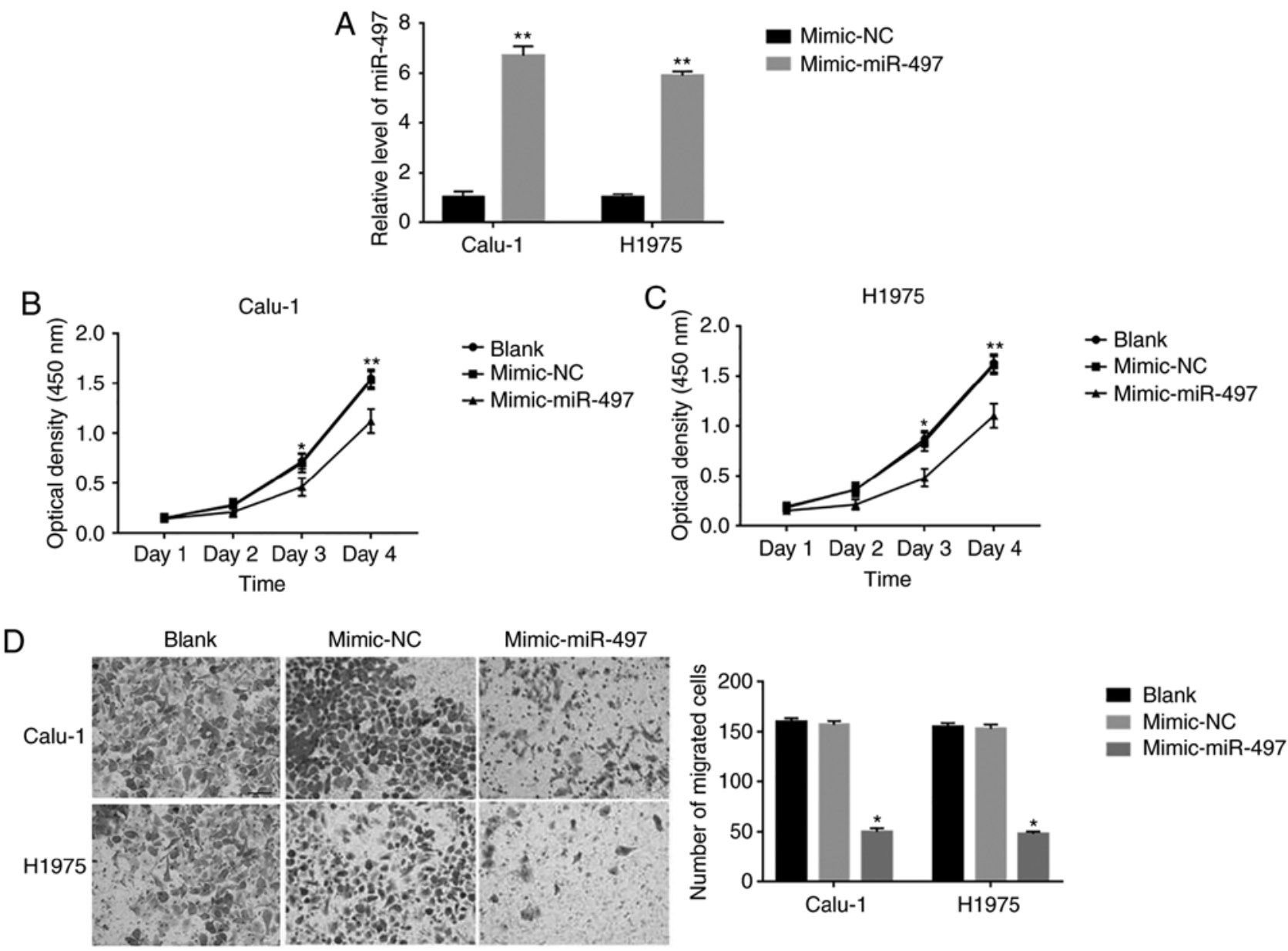

Figure 2. miR-497 overexpression decreases cell proliferation and migration in Calu-1 and H1975 cells. (A) Successful transfection of miR-497 mimic was confirmed by reverse transcription-quantitative polymerase chain reaction. (B) Cell proliferation of Calu-1 cells. (C) Cell proliferation of H1975 cells. (D) Cell migration images and quantitative histogram of Calu-1 and $\mathrm{H} 1975$ cells (x200 magnification). ${ }^{* *} \mathrm{P}<0.01$, compared with mimic-NC. NC, negative control; miR, microRNA.

lines was also examined, and the results demonstrated that KDR and miR-497 were significantly decreased in H1299 and H1975 cells, compared with in other cell lines, respectively (Fig. 1B; P<0.05). Additionally, H1975 and Calu-1 cell lines with reduced expression of miR-497 were further employed for the following experiments.

miR-497 overexpression decreases cell proliferation and migration in Calu-1 and H1975 cells. In order to observe the effects of miR-497 on the cell behavior of Calu-1 and H1975 cells, miR-497 overexpression was performed on Calu-1 and H1975 cells. As depicted in Fig. 2A, a 6.7 and 5.9 times expression level increase was exhibited in miR-497-mimic transfected Calu-1 and H1975 cells, compared with mimic-NC-transfected cells, respectively. For cell proliferation, a significantly decreased cell proliferation was observed on days 3 and 4 in miR-497-mimic-transfected Calu-1 and H1975 cells, compared with mimic-NC transfected cells (Fig. 2B and C). Furthermore, a significantly decreased cell invasion was also observed in miR-497-mimic-transfected Calu-1 (50 \pm 3.5 vs. $157 \pm 3.7 ; \mathrm{P}<0.001)$ and $\mathrm{H} 1975(48 \pm 2.4$ vs. $153 \pm 4.2 ; \mathrm{P}<0.001)$ cells, compared with mimic-NC-transfected cells (Fig. 2D). These results indicated that miR-497 overexpression decreases cell proliferation and migration in Calu-1 and H1975 cells.
miR-497 overexpression increases cell apoptosis and radiosensitivity in Calu-1 and H1975 cells. The effects of miR-497 overexpression on the cell apoptosis and radiosensitivity in Calu-1 and $\mathrm{H} 1975$ cells was further evaluated. The results demonstrated that there was significantly increased cell apoptosis following 2 Gy radiation in miR-497-mimic-transfected Calu-1 and H1975 cells, compared with mimic-NC-transfected cells (Fig. 3A; $\mathrm{P}<0.001)$. Furthermore, the clone formation was consistently decreased after 2, 4, 6 and 8 Gy radiation, and the sensitization enhancement ratio in Calu-1 and H1975 were 1.48 and 1.58, respectively (Fig. 3B and C), and miR-497 significantly increased the radiosensitivity of these cells. These results indicated the enhanced radiosensitivity following miR-497 transfection.

miR-497 affects the cell behavior of Calu-1 and H1975 via targeting KDR. In order to investigate the mechanism of miR-497 in NSCLC cell lines Calu-1 and H1975, TargetScan was searched and it was determined that KDR may be the potential target gene of miR-497 (Fig. 4A). Subsequently the KDR wild-type and mutated plasmids were constructed, and a luciferase assay was performed to confirm its interaction with miR-497. The results demonstrated that miR-497-mimic transfection significantly decreases luciferase activity in KDR wild-type, compared with mimic-NC transfected cells, while 

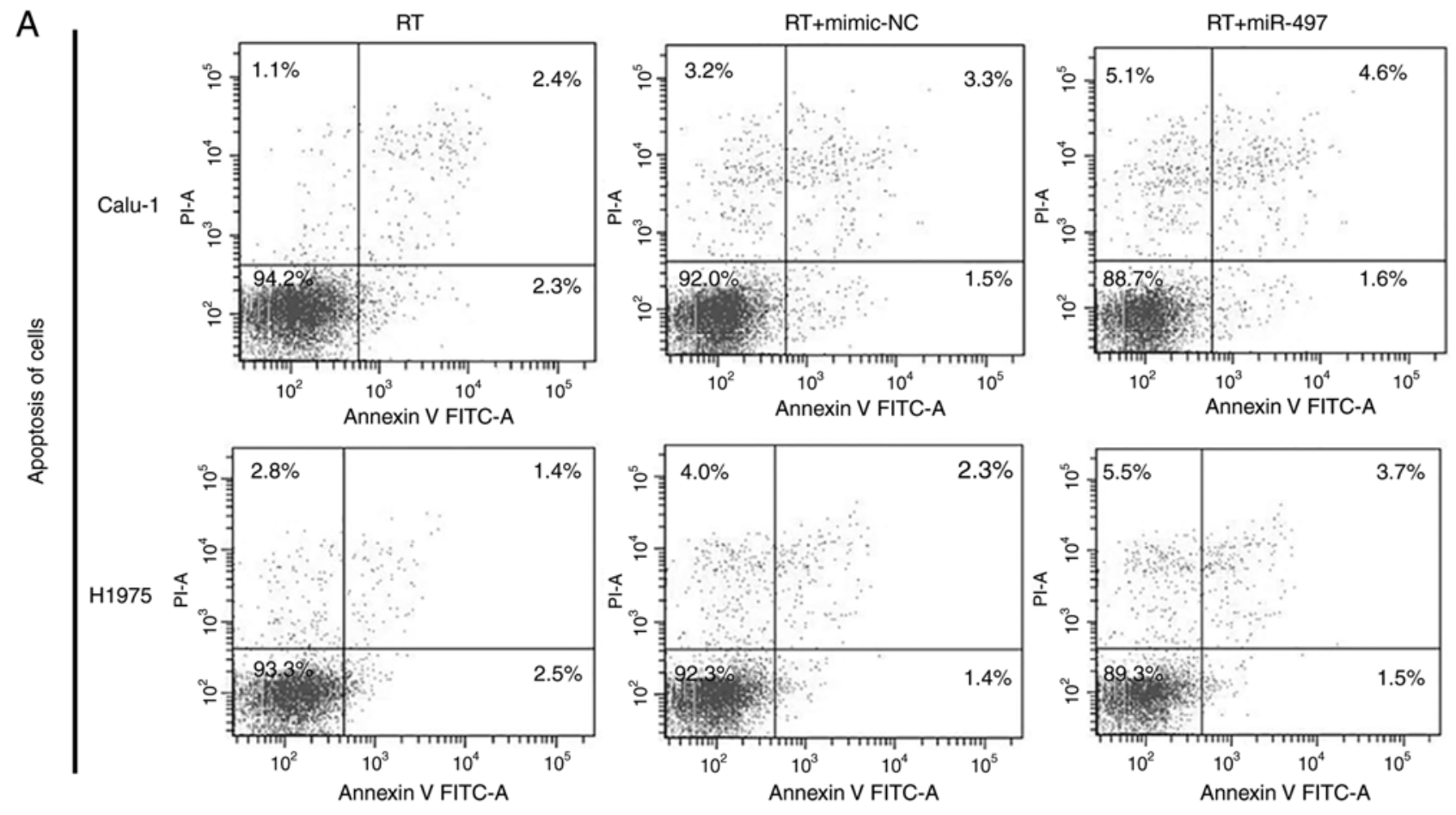

Annexin-V FITC
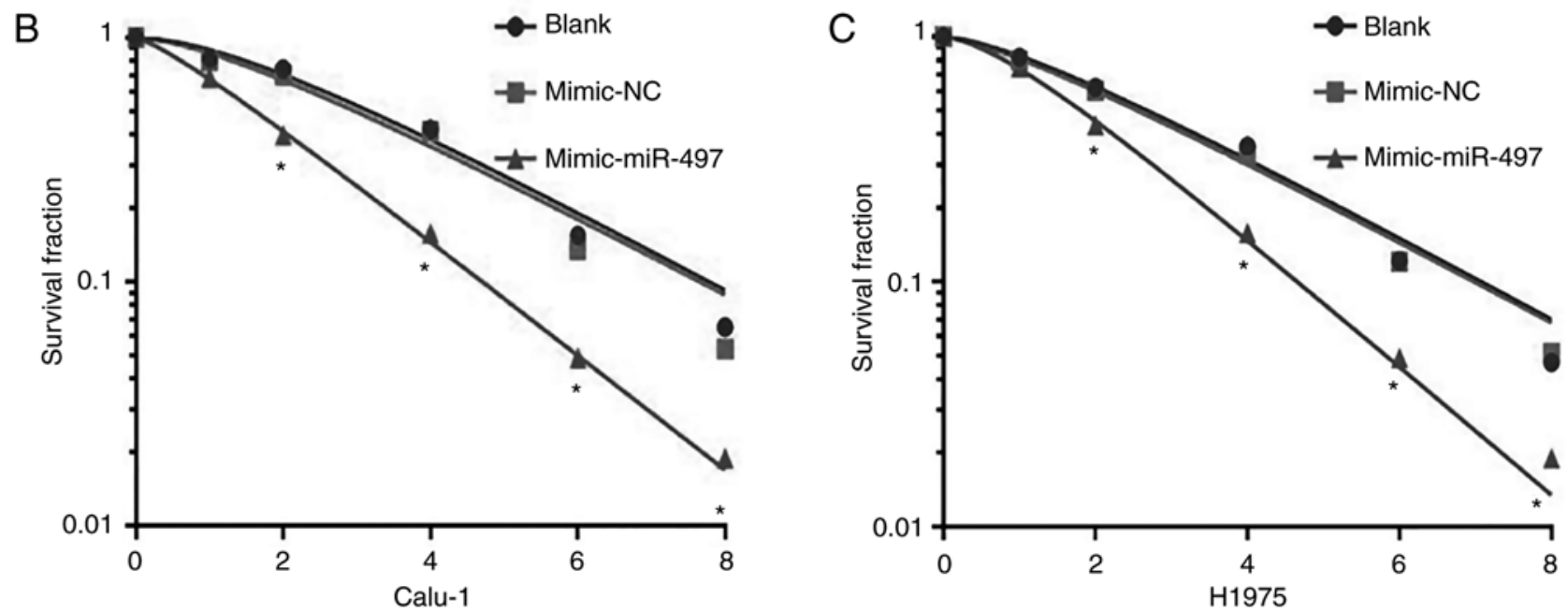

Figure 3. miR-497 overexpression increases cell apoptosis and radiosensitivity in Calu-1 and H1975 cells. (A) Flow cytometry analysis of apoptosis of lung cancer cells stained by Annexin V and Propidium Iodide. An increased cell apoptosis ratio, as depicted through the percentage number, was determined in the right upper and right lower quadrant in miR-497-overexpressed cells, compared with mimic-NC treated control cells. (B) Clone formation of Calu-1 cells. (C) Clone formation of H1975 cells. miR, microRNA; NC, negative control; RT, radiotherapy.

no difference was determined on the cells transfected with KDR mutated plasmid following miR-497-mimic or mimic-NC transfection (Fig. 4B). Furthermore, it was determined that there is significantly decreased expression of KDR in miR-497-mimic-transfected Calu-1 and H1975 cells, compared with mimic-NC-transfected cells (Fig. 4C). Additionally, it was also observed that there is a significantly decreased level of VEGFR2 in miR-497-mimic-transfected Calu-1 and H1975 cells, compared with mimic-NC-transfected cells (Fig. 4D).

miR-497 decreases tumor growth in vivo. Due the tumor-promoting effects of miR-497 observed in vitro, ectopic tumor nude mice models were established to test the effects of miR-497 in vivo. As depicted in Fig. 5, miR-497 knockdown human lung squamous carcinoma cell line Calu-1 exhibited a decreased level of tumor growth in vivo according to the tumor size and weight. These results indicated that miR-497 acts as a negative regulator on tumor growth in vivo.

\section{Discussion}

In the present study, it was demonstrated that miR-497 was downregulated in NSCLC specimens, compared with the adjacent normal tissues. Furthermore, an in vitro assay also verified 
A

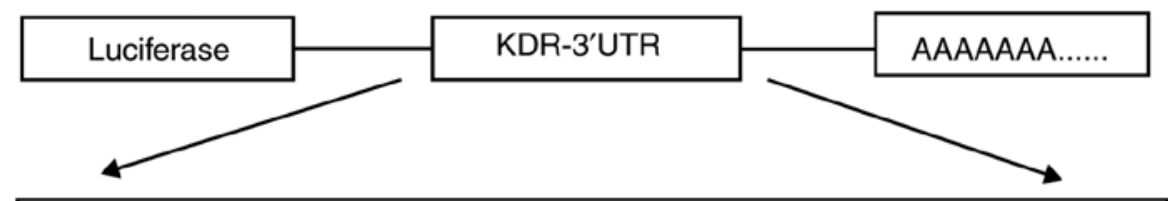

- KDR wt 3'UTR $5^{\prime}$...CAUCACAUGAGAGG UGCUGCUC... | || || |

- miR-497 $3^{\prime}$ UGUUUGGUGUCAC ACGACGAC

- KDRMt 3'UTR $5^{\prime}$... CAUCACAUGAGAGG ACGACGAC...
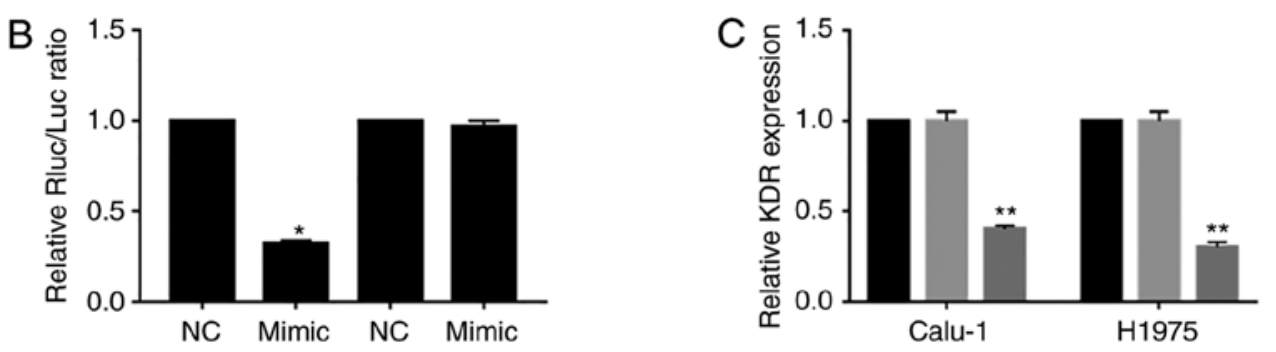

Blank

Mimic-NC

Mimic-miR-497
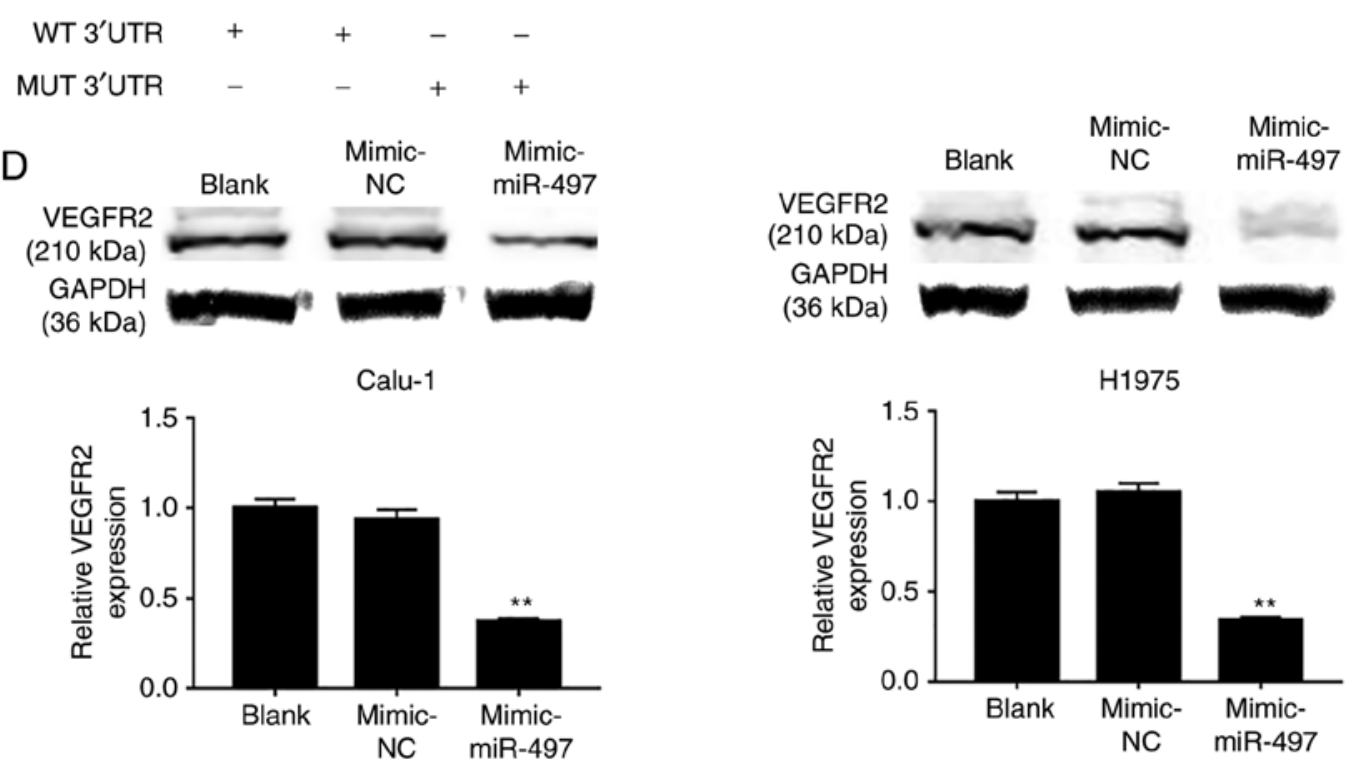

Figure 4. miR-497 affects the cell behavior of Calu-1 and H1975 via targeting KDR. (A) Schematic figure regarding the interaction between miR-497 and KDR, which was confirmed by the TargetScan tool. A mutated KDR was also exhibited for mutated luciferase plasmid construction. (B) Luciferase reporter gene analysis the interaction between miR-497 and KDR in Calu-1 cells. (C) Luciferase reporter gene analysis the interaction between miR-497 and KDR in H1975 cells. (D) Western-blotting examination of the VEGFR2 level in Calu-1 and H1975 cells. ${ }^{*} \mathrm{P}<0.05$; and ${ }^{* *} \mathrm{P}<0.01$, compared with mimic-NC. NC, negative control; miR, microRNA; UTR, untranslated region; WT, wild type; MUT, mutated; KDR, kinase insert domain receptor; VEGFR2, vascular endothelial growth factor receptor 2 .

that miR-497 overexpression could inhibit cell proliferation and invasion, and promote cell apoptosis and radiosensitivity. Additionally, KDR was also confirmed as the direct target gene of miR-497. Based on our previous study $(24,27)$, the data in the present study indicated the regulatory role of miR-497 on malignant behavior of lung cancer growth and metastasis via targeting KDR, which may serve as a malignant phenotype prediction molecule in NSCLC.

miR-497 is a common exhibited miRNA in all human organs and tissues, including breast, lung, liver and blood $(28,29)$. Dysregulated levels of miR-497 were firstly observed in miRNA expression profile results of human breast cancer, and significantly downregulated miR-497 level was then confirmed in breast cancer tissues (30). Further studies also confirmed downregulated levels of miR-497 in multiple malignant types of cancer, including gastrointestinal cancer types, thoracic cancer, reproduction cancer, and head and neck cancer types (31-33), and miR-497 served as an inhibitory regulator in the development and progression of cancer (31-33). In the present study, by collecting NSCLC tissues, it was determined that there is significantly downregulated miR-497 expression in NSCLC tissues, compared with normal tissues, indicating the possible role of miR-497 in NSCLC. 
A

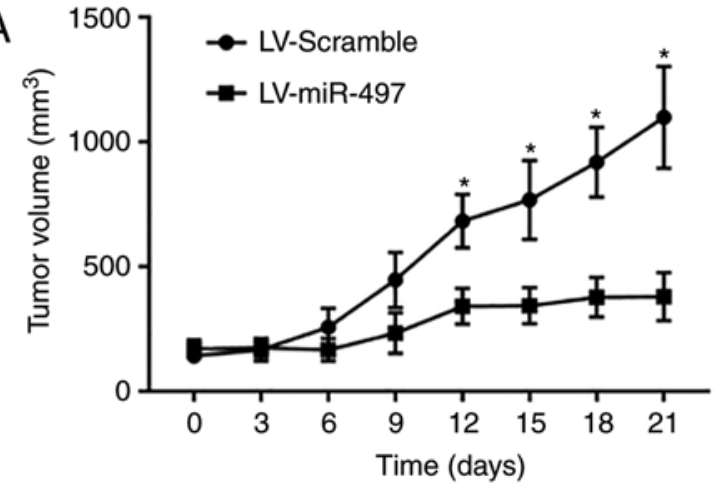

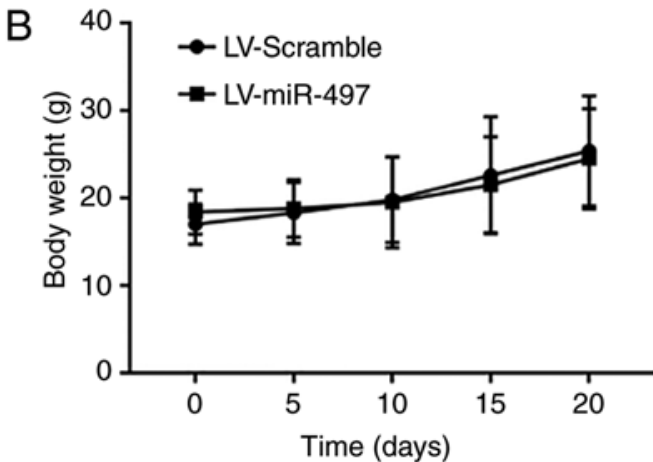

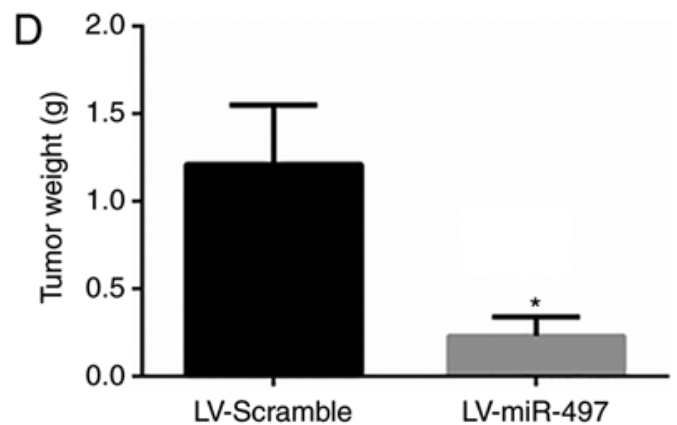

Figure 5. miR-497 overexpression in Calu-1cells decreases tumor growth in vivo. (A) Significantly decreased tumor size was observed in miR-497 overexpressed cell lines, compared with the control cell line. Time course monitoring of the tumor size. Significantly decreased tumor size was observed at days 12-21. (B) No significant difference was determined for the body weight between mice transplanted with miR-497-overexpressed and scramble-control cell lines. (C) Images of lung cancer tumor size from different groups of xenografts at day 21. Scale bar, $2 \mathrm{~cm}$. (D) Quantification of tumor weight at day 21. Significantly decreased tumor size and weight were determined in mice transplanted with miR-497-overexpressed cells, compared with the scramble-control cell line. ${ }^{*} \mathrm{P}<0.05$, compared with LV-Scramble. miR, microRNA.

According to previous studies, abnormal expression levels of miR-497 are involved in cancer progression, including proliferation, invasion, cell cycle regulation, apoptosis and angiogenesis $(34,35)$. Furthermore, multiple studies supported the observation of miR-497 as a tumor suppressor. For example, miR-497 overexpression in breast cancer could suppress tumor proliferation and invasion via target gene downregulation (36). The in vivo and in vitro system of liver cancer also confirmed that the upregulation of miR-497 could inhibit angiogenesis and metastasis of liver cancer (37). In contrast, miR-497 inhibitor transfection could result in tumor growth and proliferation promotion effects in cancer (38). $\mathrm{Xu}$ et al (39) determined that the expression levels of miR-497 were reduced in chemotherapy-resistant ovarian cancer cells and tumor tissues due to hypermethylation of the miR-497 promoter. However, Lan et al (40) determined that miR-497 is overexpressed in glioma and that hypoxia can induce the expression of miR-497 at the transcriptional level by binding with the hypoxia response element in the promoter. They also indicated that ectopic overexpression of miR-497 promotes chemotherapy resistance in glioma cells by targeting programmed cell death 4, a tumor suppressor that is involved in apoptosis (40). Discrepancies regarding the role of miR-497 in cancer may result from the heterogeneous of miR-497 expression and different tumor microenvironment. In NSCLC, miR-497 was demonstrated to exhibit a previously unappreciated role in the suppression of VEGF-A-mediated NSCLC cancer cell growth and invasion (31). Consistent with previous study, in the present study, it was demonstrated that miR-497 overexpression could inhibit cell proliferation and invasion, promote cancer cell apoptosis and decrease cell clone formation following radiation treatment.

A single miRNA can target hundreds of mRNAs, and a series of target genes were determined to exert inhibitory effects by interacting with miR-497, including cyclin E1, B-cell lymphoma, insulin like growth factor 1 receptor and VEGFR2 (41-44). In the present study, the dual luciferase reporter gene assay revealed that KDR was the direct target gene for miR-497 at the 45-51 sites of 3'UTR, which is consistent with results in kidney cancer (45). It was also determined that miR-497 could downregulate KDR mRNA and protein levels of VEGFR2 (45). According to a previous study by Shi et al (46), VEGFR2knockdown could result in decreased survival fraction of the lung cancer cells, angiogenesis and migration during carcinogenesis. Furthermore, the direct blockage of VEGFR2 by monoclonal antibody ramucirumab could result in clinical benefits, compared with docetaxel only-treated patients, in terms of progression-free survival, objective response rate and disease control rate in Asian patients with NSCLC (47). Additionally, ramucirumab was well tolerated with manageable toxicity (47). Furthermore, Ding et al (48) examined the expression of KDR in NSCLC tissues, and they determined that the positive immunostaining rate for VEGFR2 was 58\%. Levels of VEGFR2 in lung tumors were significantly increased compared with in the control tissue $\left(\chi^{2}=11.22 ; \mathrm{P}=0.001\right)$. Statistically significant correlations were observed with histological grade, clinical TNM stage and the lymph node status $(\mathrm{P}<0.05)$, but not age, sex or pathology type $(\mathrm{P}>0.05)$ between NSCLC tissues 
and control tissues. Furthermore, significant decreased overall survival time and progression-free survival time were observed between the groups with increased VEGFR2 expression and those with reduced expression $(\mathrm{P}<0.05)$. Reduced levels of VEGFR2 in lung cancer tissues were consistent with the results that miR-497 directly targeted KDR and downregulated KDR expression, thereby resulting in inhibition of malignant behaviors of NSCLC cells. In our previous study, it was also determined that KDR silence and pharmacological inhibition of VEGFR2 could result in the inhibition of cell proliferation, invasion and radiosensitivity enhancement (27), and these results were consistent with the results in the present study regarding miR-497.

Furthermore, since this paper did not perform a correlation analysis between the miR-497 expression and clinical characteristics, the role of miR-497 on prognosis of the patients could not be evaluated. Additionally, the sample size may be insufficient to elucidate the role of miR-497 in lung cancer according to previous studies [17 in the study by Devery et al (49); and 24 in the study by Zhu et al (50)].

In conclusion, the present study demonstrated that miR-497 was downregulated in NSCLC specimens and it served as a tumor suppressor to inhibit cancer cell proliferation and invasion, and increase radiosensitivity via targeting KDR. The regulatory role between miR-497 and KDR may provide novel insight for lung cancer progression control, radiotherapy sensitivity enhancement and target therapy strategy.

\section{Acknowledgements}

The authors would like to thank Professor Aldo Pinto from Department of Pharmacy, School of Pharmacy, University of Salerno (Fisciano, Italy) for his critical reading of the present manuscript.

\section{Funding}

The present study was supported by the National Natural Science Foundation of China (grant no. 81472792), the Natural Science Foundation of Jiangsu Province, China (grant no. BK20151279), the Youth Talent Foundation of Lianyungang First People's Hospital (grant no. QN140202), the Science and Technology Development Program of Lianyungang City(grant no. ZD1404), the '521 Project' Foundation of Lianyungang City, the '333 Project' Foundation of Jiangsu Province (grant no. BRA2016301). the Science and Technology Project Foundation of Suzhou (grant no. SYS201504).

\section{Availability of data and materials}

The datasets used and/or analyzed during the current study are available from the corresponding author on reasonable request.

\section{Authors' contributions}

$\mathrm{YX}, \mathrm{CH}, \mathrm{LianL}$ and $\mathrm{XJ}$ conceived and designed the experiments. YX, CH, LL, KH, LW, YQ, LiangL and LijunL performed the experiments. YX, CH, LianL, KH, LW, YQ, LiangL, LijunL and XJ analyzed the data. YX, CH, LianL, KH, LW, YQ, LiangL, LijunL and XJ contributed reagents, materials and analysis tools. YX, CH, LianL and XJ contributed to the writing of the manuscript. All authors read and approved the final manuscript.

\section{Ethics approval and consent to participate}

The study protocol was approved by the Ethics Committee of the First People's Hospital of Lianyungang (Lianyungang, China). Written informed consent was obtained from the participating individuals.

\section{Patient consent for publication}

Written informed consent was obtained.

\section{Competing interests}

The authors declare that they have no competing interests.

\section{References}

1. Torre LA, Bray F, Siegel RL, Ferlay J, Lortet-Tieulent J and Jemal A: Global cancer statistics, 2012. CA Cancer J Clin 65: 87-108, 2015.

2. Wang S, Wang X, Zhou Q, Xu Y, Xia W, Xu W, Ma Z, Qiu M, You R, Xu L and Yin R: Stereotactic ablative radiotherapy versus lobectomy for stage I non-small cell lung cancer: A systematic review. Thorac Cancer 9: 337-347, 2018

3. Ferlay J, Soerjomataram I, Dikshit R, Eser S, Mathers C, Rebelo M, Parkin DM, Forman D and Bray F: Cancer incidence and mortality worldwide: Sources, methods and major patterns in GLOBOCAN 2012. Int J Cancer 136: E359-E386, 2015.

4. Hassan O, Ahmad A, Sethi S and Sarkar FH: Recent updates on the role of microRNAs in prostate cancer. J Hematol Oncol 5: 9, 2012.

5. Du L and Pertsemlidis A: microRNA regulation of cell viability and drug sensitivity in lung cancer. Expert Opin Biol Ther 12: 1221-1239, 2012.

6. Acunzo M, Visone R, Romano G, Veronese A,Lovat F, Palmieri D, Bottoni A, Garofalo M, Gasparini P, Condorelli G, et al: miR-130a targets MET and induces TRAIL-sensitivity in NSCLC by downregulating miR-221 and 222. Oncogene 31: 634-642, 2012.

7. Jeon HS, Lee SY, Lee EJ, Yun SC, Cha EJ, Choi E, Na MJ, Park JY, Kang J and Son JW: Combining microRNA-449a/b with a HDAC inhibitor has a synergistic effect on growth arrest in lung cancer. Lung Cancer 76: 171-176, 2012.

8. Liu X, Sempere LF, Guo Y, Korc M, Kauppinen S, Freemantle SJ and Dmitrovsky E: Involvement of microRNAs in lung cancer biology and therapy. Transl Res 157: 200-208, 2011.

9. Itesako T, Seki N, Yoshino H, Chiyomaru T, Yamasaki T, Hidaka H, Yonezawa T, Nohata N, Kinoshita T, Nakagawa M and Enokida $\mathrm{H}$ : The microRNA expression signature of bladder cancer by deep sequencing: The functional significance of the miR-195/497 cluster. PLoS One 9: e84311, 2014.

10. Li W, Jin X, Deng X, Zhang G, Zhang B and Ma L: The putative tumor suppressor microRNA-497 modulates gastric cancer cell proliferation and invasion by repressing eIF4E. Biochem Biophys Res Commun 449: 235-240, 2014.

11. Xie Y, Wei RR, Huang GL, Zhang MY, Yuan YF and Wang HY: Checkpoint kinase 1 is negatively regulated by miR-497 in hepatocellular carcinoma. Med Oncol 31: 844, 2014.

12. Du M, Shi D, Yuan L, Li P, Chu H, Qin C, Yin C, Zhang Z and Wang M: Circulating miR-497 and miR-663b in plasma are potential novel biomarkers for bladder cancer. Sci Rep 5: 10437, 2015.

13. Liu L, Zheng W, Song Y, Du X, Tang Y, Nie J and Han W: miRNA-497 enhances the sensitivity of colorectal cancer cells to neoadjuvant chemotherapeutic drug. Curr Protein Pept Sci 16: 310-315, 2015.

14. Zhao WY, Wang Y, An ZJ, Shi CG, Zhu GA, Wang B, Lu MY, Pan CK and Chen P: Downregulation of miR-497 promotes tumor growth and angiogenesis by targeting HDGF in non-small cell lung cancer. Biochem Biophys Res Commun 435: 466-471, 2013. 
15. Shibuya M: Vascular endothelial growth factor (VEGF) and its receptor (VEGFR) signaling in angiogenesis: A crucial target for anti- and pro-angiogenic therapies. Genes Cancer 2: 1097-1105, 2011.

16. Wang J, Chen J, Guo Y, Wang B and Chu H: Strategies targeting angiogenesis in advanced non-small cell lung cancer. Oncotarget 8: 53854-53872, 2017.

17. Guo L, Zhang F, Cai Y and Liu T: Expression profiling of integrins in lung cancer cells. Pathol Res Pract 205: 847-853, 2009.

18. Brower M, Carney DN, Oie HK, Gazdar AF and Minna JD Growth of cell lines and clinical specimens of human non-small cell lung cancer in a serum-free defined medium. Cancer Res 46 798-806, 1986

19. Suzuki S, Takahashi T, Nakamura S, Koike K, Ariyoshi Y, Takahashi T and Ueda R: Alterations of integrin expression in human lung cancer. Jpn J Cancer Res 84: 168-174, 1993.

20. Banks-Schlegel SP, Gazdar AF and Harris CC: Intermediate filament and cross-linked envelope expression in human lung tumor cell lines. Cancer Res 45: 1187-1197, 1985.

21. Hu X, Shi S, Wang H, Yu X, Wang Q, Jiang S, Ju D, Ye L and Feng M: Blocking autophagy improves the anti-tumor activity of afatinib in lung adenocarcinoma with activating EGFR mutations in vitro and in vivo. Sci Rep 7: 4559, 2017.

22. Chen QY, Wu LJ, Wu YQ, Lu GH, Jiang ZY, Zhan JW, Jie Y and Zhou JY: Molecular mechanism of trifluoperazine induces apoptosis in human A549 lung adenocarcinoma cell lines. Mol Med Rep 2: 811-817, 2009.

23. Giaccone G, Battey J, Gazdar AF, Oie H, Draoui M and Moody TW: Neuromedin B is present in lung cancer cell lines. Cancer Res 52 (Suppl 9): S2732-S2736, 1992.

24. Liu Y, Qiao Y, Hu C, Liu L, Zhou L, Liu B, Chen H and Jiang X: VEGFR2 inhibition by RNA interference affects cell prolif eration, migration, invasion, and response to radiation in Calu-1 cells. Clin Transl Oncol 18: 212-219, 2016.

25. Ji Y, Strawn TL, Grunz EA, Stevenson MJ, Lohman AW, Lawrence DA and Fay WP: Multifaceted role of plasminogen activator inhibitor-1 in regulating early remodeling of vein bypass grafts. Arterioscler Thromb Vasc Biol 31: 1781-1787, 2011.

26. Ferdowsian HR and Beck N: Ethical and scientific considerations regarding animal testing and research. PLoS One 6: e24059, 2011

27. Liu L, Qiao Y, Hu C, Liu Y, Xia Y, Wang L, Liu B, Chen H and Jiang $\mathrm{X}$ : Endostatin exerts radiosensitizing effect in non-small cell lung cancer cells by inhibiting VEGFR2 expression. Clin Transl Oncol 18: 18-26, 2016.

28. Ruan K, Fang X and Ouyang G: MicroRNAs: Novel regulators in the hallmarks of human cancer. Cancer Lett 285: 116-126, 2009.

29. Rapa I, Votta A, Felice B, Righi L, Giorcelli J, Scarpa A, Speel EJ, Scagliotti GV, Papotti $M$ and Volante $M$ : Identification of MicroRNAs differentially expressed in lung carcinoid subtypes and progression. Neuroendocrinology 101: 246-255, 2015.

30. Yan LX, Huang XF, Shao Q, Huang MY, Deng L, Wu QL, Zeng YX and Shao JY: MicroRNA miR-21 overexpression in human breast cancer is associated with advanced clinical stage, lymph node metastasis and patient poor prognosis. RNA 14: 2348-2360, 2008.

31. Gu A, Lu J, Wang W, Shi C, Han B and Yao M: Role of miR-497 in VEGF-A-mediated cancer cell growth and invasion in non-small cell lung cancer. Int J Biochem Cell Biol 70: 118-125, 2016.

32. Wang S, Mo Y, Midorikawa K, Zhang Z, Huang G, Ma N, Zhao W, Hiraku Y, Oikawa S and Murata M: The potent tumor suppressor miR-497 inhibits cancer phenotypes in nasopharyngeal carcinoma by targeting ANLN and HSPA4L. Oncotarget 6 : 35893-35907, 2015.

33. Liu A, Huang C, Cai X, Xu J and Yang D: Twist promotes angiogenesis in pancreatic cancer by targeting miR-497/VEGFA axis. Oncotarget 7: 25801-25814, 2016.

34. Yang G, Xiong G, Cao Z, Zheng S, You L, Zhang T and Zhao Y: miR-497 expression, function and clinical application in cancer Oncotarget 7: 55900-55911, 2016.
35. Zhao X, Zhao Z, Xu W, Hou J and Du X: Down-regulation of miR-497 is associated with poor prognosis in renal cancer. Int J Clin Exp Pathol 8: 758-764, 2015.

36. Li D, Zhao Y, Liu C, Chen X, Qi Y, Jiang Y, Zou C, Zhang X, Liu S, Wang X, et al: Analysis of miR-195 and miR-497 expression, regulation and role in breast cancer. Clin Cancer Res 17: $1722-1730,2011$

37. Yan JJ, Zhang YN, Liao JZ, Ke KP, Chang Y, Li PY, Wang M, Lin JS and He XX: miR-497 suppresses angiogenesis and metastasis of hepatocellular carcinoma by inhibiting VEGFA and AEG-1. Oncotarget 6: 29527-29542, 2015.

38. Wei C, Luo Q, Sun X, Li D, Song H, Li X, Song J, Hua K and Fang L: MicroRNA-497 induces cell apoptosis by negatively regulating Bcl-2 protein expression at the posttranscriptional level in human breast cancer. Int J Clin Exp Pathol 8: 7729-7739, 2015.

39. Xu S, Fu GB, Tao Z, OuYang J, Kong F, Jiang BH, Wan X and Chen K: miR-497 decreases cisplatin resistance in ovarian cancer cells by targeting mTOR/P70S6K1. Oncotarget 6: 26457-26471, 2015.

40. Lan J, Xue Y, Chen H, Zhao S, Wu Z, Fang J, Han C and Lou M: Hypoxia-induced miR-497 decreases glioma cell sensitivity to TMZ by inhibiting apoptosis. FEBS Lett 588: 3333-3339, 2014.

41. Han Z, Zhang Y, Yang Q, Liu B, Wu J, Zhang Y, Yang C and Jiang Y: miR-497 and miR-34a retard lung cancer growth by co-inhibiting cyclin E1 (CCNE1). Oncotarget 6: 13149-13163, 2015.

42. Shen L, Li J, Xu L, Ma J, Li H, Xiao X, Zhao J and Fang L: miR-497 induces apoptosis of breast cancer cells by targeting Bcl-w. Exp Ther Med 3: 475-480, 2012.

43. Xu JW, Wang TX, You L, Zheng LF, Shu H, Zhang TP and Zhao YP: Insulin-like growth factor 1 receptor (IGF-1R) as a target of miR-497 and plasma IGF-1R levels associated with TNM stage of pancreatic cancer. PLoS One 9: e92847, 2014.

44. Zhu W, Zhu D, Lu S, Wang T, Wang J, Jiang B, Shu Y and Liu P: miR-497 modulates multidrug resistance of human cancer cell lines by targeting BCL2. Med Oncol 29: 384-391, 2012.

45. Pengcheng S, Ziqi W, Luyao Y, Xiangwei Z, Liang L, Yuwei L, Lechen L and Wanhai X: MicroRNA-497 suppresses renal cell carcinoma by targeting VEGFR-2 in ACHN cells. Biosci Rep 37: BSR20170270, 2017.

46. Shi L, Zhang S, Wu H, Zhang L, Dai X, Hu J, Xue J, Liu T, Liang $\mathrm{Y}$ and $\mathrm{Wu} \mathrm{G}$ : miR-200c increases the radiosensitivity of non-small-cell lung cancer cell line A549 by targeting VEGF-VEGFR2 pathway. PLoS One 8: e78344, 2013.

47. Hosomi Y, Yoh K, Kasahara K, Yamada K, Takahashi T, Hida TTK, Yoshioka H, Kato T, Takeda K, Nishio M, et al: Docetaxel + ramucirumab (DR) versus docetaxel + placebo (D) as second-line treatment for advanced non-small cell lung cancer (NSCLC): A randomized, phase II, double-blind, multicenter trial in Japan. J Clin Oncol 33: 8054-8054, 2015.

48. Ding M, Liu L, Hu C, Liu Y, Qiao Y and Jiang X: Expression of VEGFR2 and NRP-1 in non-small cell lung cancer and their clinical significance. Chin J Cancer Res 26: 669-677, 2014.

49. Devery AM, Wadekar R, Bokobza SM, Weber AM, Jiang Y and Ryan AJ: Vascular endothelial growth factor directly stimulates tumour cell proliferation in non-small cell lung cancer. Int J Oncol 47: 849-856, 2015.

50. Zhu J, Zeng Y, Li W, Qin H, Lei Z, Shen D, Gu D, Huang JA and Liu Z: CD73/NT5E is a target of miR-30a-5p and plays an important role in the pathogenesis of non-small cell lung cancer. Mol Cancer 16: 34, 2017. 